\title{
Disruption of Layers 3 and 4 during Development Results in Altered Thalamocortical Projections in Ferret Somatosensory Cortex
}

\author{
Stephen C. Noctor, ${ }^{1}$ Sidney L. Palmer, ${ }^{2}$ Debra F. McLaughlin, ${ }^{1,2}$ and Sharon L. Juliano ${ }^{1,2}$ \\ ${ }^{1}$ Program in Neuroscience and 2 Department of Anatomy and Cell Biology, Uniformed Services University of the Health \\ Services, Bethesda, Maryland 20814
}

The precision of projections from dorsal thalamus to neocortex are key toward understanding overall cortical organization and function. To identify the significance of layer 4 cells in receiving the bulk of thalamic projections in somatosensory cortex, we disrupted layer 4 genesis and studied the effect on thalamic terminations in ferrets. Second, we ascertained the result of layer 4 disruption on functional responses and topographic organization. Methylazoxy methanol (MAM) was injected into pregnant ferrets on embryonic day 33 (E33), when most layer 4 neurons of somatosensory cortex are generated. This treatment resulted in dramatic reduction in the thickness of targeted layer 4. E38 MAM treatment was used as a control, when layer 2-3 neurons are generated. The projections of ventrobasal thalamus into somatosensory cortex were studied using Dil injections. We found only subtle differences between groups (normal, E33, or E38 MAM-treated) in the thalamic afferent pattern

The precise patterning of thalamic growth into specific cortical layers is primarily responsible for the explicit function and structure of the cerebral cortex. Each cytoarchitectonic field is formed partly by its intrinsic architecture and partly by specific interactions between thalamus and cortex. The mechanisms directing growth of thalamic axons into their proper site of termination have been a subject of interest for a number of years (Hubener et al., 1995; Mann et al., 1998; Molnar et al., 1998). In most primary sensory neocortical areas, layer 4 is a primary recipient of specific thalamic nuclei afferents, although layer 3 also receives a substantial portion of thalamic projections. A number of recent studies attempt to define factors that are "special" about layer 4 and why most thalamic fibers stop there (Molnar et al., 1991, 1999; Gotz et al., 1992; Bicknese et al., 1994; Hubener et al., 1995; Miller et al., 1995; Yamamoto et al., 1997; Molnar and Blakemore, 1999).

To further define the influence of layer 4 on the ultimate position and function of thalamic terminations, we disrupted the development of this layer using injections of methylazoxy methanol (MAM) during gestation of the ferret. Cells destined for layer 4 of ferret somatosensory cortex are generated on embryonic days 33 (E33) and E34 (Noctor et al., 1997). MAM selectively prevents the production of postmitotic neurons by stopping the synthesis of new DNA during S-phase. Cells dividing at the

\footnotetext{
Received Sept. 14, 2000; revised Jan. 9, 2001; accepted Feb. 5, 2001.

This work was supported by United States Public Health Service Grant RO1 NS24014. We thank Donna Tatham for expert technical assistance.

S.C.N. and S.L.P. contributed equally to this manuscript.

Correspondence should be addressed to Sharon L. Juliano, Department of Anatomy and Cell Biology, 4301 Jones Bridge Road, Bethesda, MD 20814. E-mail: sjuliano@usuhs.mil.

Copyright (C) 2001 Society for Neuroscience $\quad 0270-6474 / 01 / 213184-12 \$ 15.00 / 0$
}

on postnatal day 1 (P1) and P7. On P14, thalamic terminations distribute almost equally throughout the remaining cortical layers in the E33 MAM-treated group compared with normal and E38 MAM-treated animals, in which the ventrobasal thalamus projects primarily to central layers. Electrophysiological recordings conducted on mature ferrets treated with MAM on E33 demonstrated that somatotopic organization and receptive field size are normal. These findings emphasize the importance of layer 4 in determining the normal laminar pattern of thalamic termination and suggest that, although its absence is likely to impact on complex neocortical functional responses, topographic organization does not arise from the influence of layer 4.

Key words: cerebral cortex; development; MAM; migration disorder; ventrobasal thalamus; $\mathrm{BrdU}$ time of the injection were removed from the cell cycle and prevented from influencing subsequent events in cortical development (Cattaneo et al., 1995).

Our study used ferrets, in which the generation of each neocortical layer extends for at least several days (Jackson et al., 1989; Noctor et al., 1997). In these animals, appropriately timed MAM treatments can target a specific layer while minimizing effects on the later generated cortical layers. Because layer 4 is often presumed to impart the character of sensory cortex, we also assessed functional responses and topographic organization in ferret primary somatosensory cortex. We report that interruption of layer 4 development using MAM in ferrets results in altered projections from the ventrobasal thalamus (VB). Rather than terminating in a focussed pattern in the vicinity of layer 4, VB projects diffusely to all cortical layers with many fibers terminating in upper layers. The electrophysiological responses in this region, however, were similar to those in normal somatosensory cortex.

\section{MATERIALS AND METHODS}

Experimental design. During pregnancy, ferrets were injected with MAM on either E33 or E38. In one set of experiments, the cytoarchitecture of somatosensory cortex was examined in normal and MAM-treated ferrets at birth [postnatal day 0 (P0) to P1] and when they were 3 months old. In another experimental set, brains were removed and blocked, and DiI injections were placed in the VB in normal and MAM-treated animals at different ages (P1, P7, and P14). After an appropriate period to allow for diffusion of the tracer, the brains were cut and analyzed to assess the distribution of label found in the cerebral cortex. This study focussed on projections that terminated in primary somatosensory cortex. We included for quantitative study a restricted block of cortex $\sim 200-\mu$ m-wide in a site immediately posterior and lateral to the post cruciate dimple (Fig. 1). The numbers of animals assessed for analysis of cytoarchitecture 

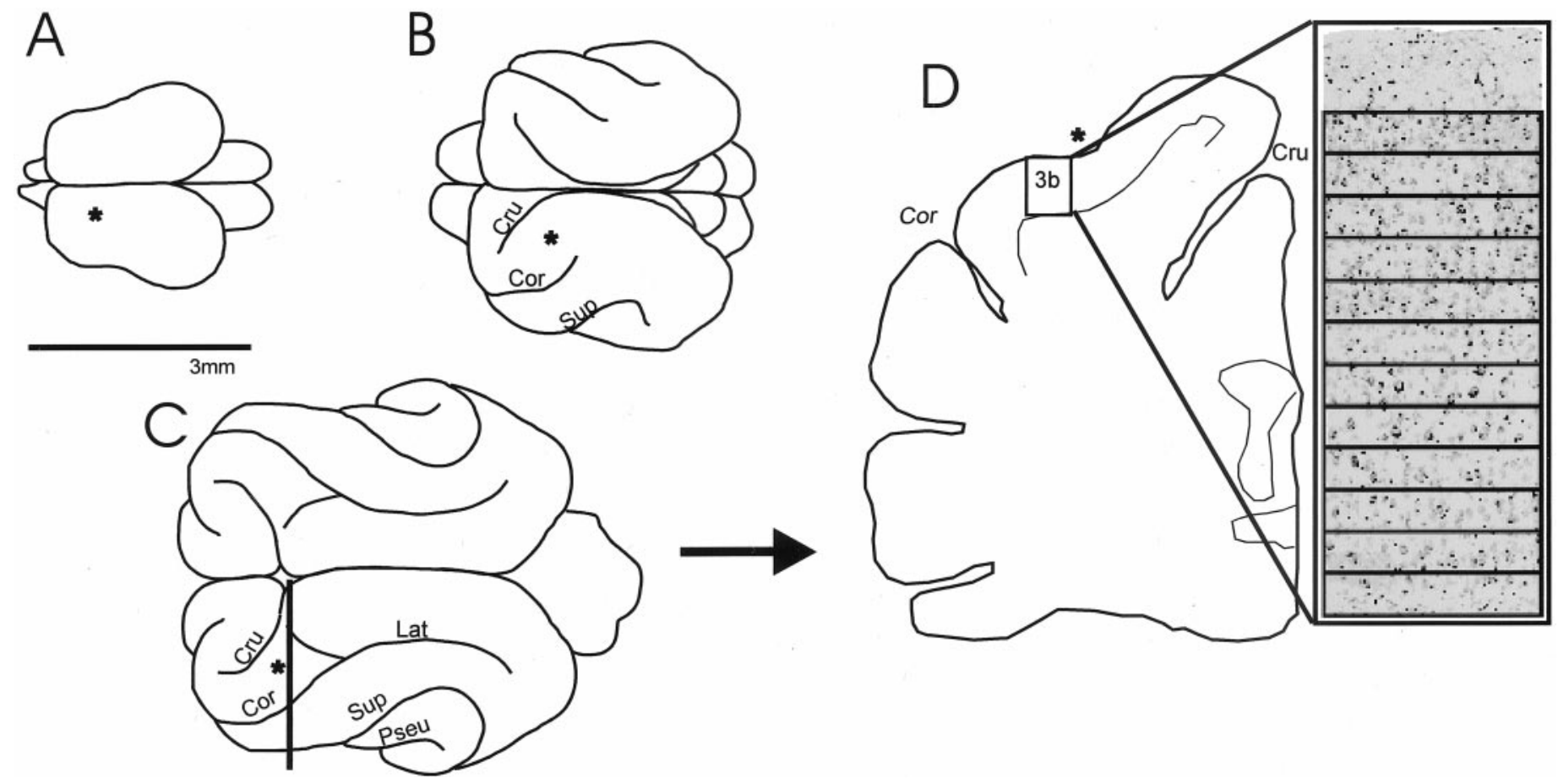

Figure 1. Drawings of ferret brains from the dorsal perspective at $\mathrm{P} 1(A), \mathrm{P} 7(B)$, and $\mathrm{P} 14(C)$. The position and plane of section used for quantitative analysis is indicated with a bar located just posterior to the post cruciate dimple (asterisk) in $C$. The position of the box in $D$ displays the approximate position of the site chosen for analysis, just lateral to the post cruciate dimple. At P1, very few sulci are visible but become more evident at P7 and P14. Primary somatosensory cortex can be identified from surface landmarks but was confirmed histologically in both normal and E33 M AM-treated ferrets. The vertical line indicating the approximate position of the region assessed for quantitative analysis is illustrated in the coronal section to the right. $D$, For quantitative analysis of the cytoarchitecture in mature animals, a region of cortex in area $3 \mathrm{~b}$ just lateral to the post cruciate dimple (asterisk) and medial to the coronal sulcus (Cor) was imaged in each section; it contained a radial sector of cortex 500- $\mu$ m-wide, extending from the top of layer 2 to the white matter (rectangle). The rectangular inset is an example of a digitized image taken from the selected region; the division of the selected region into a grid of boxes is illustrated. The block of cortex assessed for the distribution of DiI was in a similar location but slightly wider. Cru, Cruciate sulcus; Lat, lateral sulcus. Scale bar, $3 \mathrm{~mm}$.

\section{Table 1. Total number of animals used in all experiments}

Number of pregnant ferrets

$\begin{array}{lr}\text { Normal } & 11 \\ \text { E33 MAM } & 10 \\ \text { E38 MAM } & 3\end{array}$

Number of animals assessed for cytoarchitectural analysis

\begin{tabular}{lcl}
\hline & P0-P1 & 3 Months \\
\hline E33 MAM & 16 & 5 \\
E38 MAM & 4 & 4 \\
Normal & 7 & 4 \\
\hline
\end{tabular}

Number of animals analyzed after DiI injections into VB

\begin{tabular}{lllll}
\hline & E33 & P1 & P7 & P14 \\
\hline E33 MAM & & 5 & 4 & 3 \\
E38 MAM & & 4 & 3 & 2 \\
Normal & 3 & 5 & 3 & 3
\end{tabular}

and for distribution of DiI label found in the somatosensory cortex after injections in the ventrobasal thalamus are reported in Table 1.

$M A M$ and bromodeoxyuridine treatment. Pregnant ferrets (Marshall Farms, New Rose, NY) were anesthetized with 5\% halothane and $0.05 \%$ $\mathrm{N}_{2} \mathrm{O}$. An injection of methylazoxy methanol acetate $(12 \mathrm{mg} / \mathrm{kg}$; Sigma,
St. Louis, MO) was administered intraperitoneally on either E33 or E38. The MAM injections disrupted cells undergoing final mitosis that were intended for either layer 4 (E33) or layers 2-3 (E38). We determined previously the precise days of gestation for cells populating specific layers of ferret somatosensory cortex (Noctor et al., 1997). Three days after MAM treatment, three of the pregnant ferrets were treated with single intraperitoneal injections of $80 \mathrm{mg} / \mathrm{kg}$ bromodeoxyuridine (BrdU), as described previously (Noctor et al., 1997). Ferrets were closely monitored after injections to ensure proper health. Data from normal pregnant ferrets injected with BrdU on E33 and used in another study (Noctor et al., 1997) were also included for comparison purposes.

Immunohistochemistry. Ferrets were anesthetized with an overdose of pentobarbital $\mathrm{Na}(60 \mathrm{mg} / \mathrm{kg}$, i.p.) and perfused through the heart with saline, followed by $4 \%$ paraformaldehyde in $0.1 \mathrm{M}$ phosphate buffer at $\mathrm{pH}$ 7.4. Brains were removed, sunk in $20 \%$ sucrose for cryoprotection, frozen in isopentane at $-35^{\circ} \mathrm{C}$, and stored at $-80^{\circ} \mathrm{C}$. The region containing the somatosensory cortex was blocked, and sections were cut on a cryostat at $30 \mu \mathrm{m}$. In animals that received BrdU injections after MAM treatment, alternate sections were immunostained with antibodies directed against BrdU using a method modified from Miller and Nowakowski (1988). The tissue was incubated for $30 \mathrm{~min}$ in $95 \% \mathrm{EtOH}$ and $5 \%$ acetic acid, followed by $1 \mathrm{hr}$ in $2 \mathrm{~N} \mathrm{HCl}$ with $1 \mathrm{mg} / \mathrm{ml}$ pepsin at $37^{\circ} \mathrm{C}$. Sections were then rinsed with $0.1 \mathrm{M} \mathrm{PBS}$ at $\mathrm{pH} 8.5$ and incubated overnight at $4^{\circ} \mathrm{C}$ in the primary antibody (anti-BrdU; 1:20; Becton Dickinson, Cockeysville, $\mathrm{MD}$ ) in PBS, $\mathrm{pH} 7.4$, with $0.1 \%$ Triton $\mathrm{X}-100$. On the following day, standard procedures were followed for peroxidase labeling using Vector kits (Vector Elite ABC kit; Vector Laboratories, Burlingame, CA). Alternate sections were stained for identification of Nissl substance using cresyl violet.

DiI injections. At P1, P7, or P14, each ferret received an intraperitoneal injection of pentobarbital $\mathrm{Na}(50 \mathrm{mg} / \mathrm{kg})$. When insensitive to pain, the animal was transcardially perfused with saline, followed by $4 \%$ paraformaldehyde in $0.1 \mathrm{M}$ phosphate buffer. The brains were removed, blocked, 
and placed in the same fixative at $4^{\circ} \mathrm{C}$ until DiI was injected. At the time of the DiI injection, the blocks were trimmed coronally from the posterior aspect to the level of the posterior thalamus. A crystal of DiI was inserted into the ventrobasal nucleus of the thalamus under microscopic guidance using a pulled glass pipette. After an incubation period of $6-8$ weeks, each cortical hemisphere was embedded in 3\% agar and cut at 100 $\mu \mathrm{m}$ thickness in the coronal plane on a vibratome. Each slice was mounted on a gelatin-subbed slide and counterstained with a $0.2 \%$ bisbenzimide solution for $2 \mathrm{~min}$ and then coverslipped with fluorescent mounting media.

Cytoarchitectural analysis of adult somatosensory cortex. To facilitate a detailed comparison of the cytoarchitecture observed in MAM-treated and normal ferret somatosensory cortex, 3-month-old ferrets were anesthetized with an overdose of pentobarbital $\mathrm{Na}(60 \mathrm{mg} / \mathrm{kg}$, i.p.) and then perfused through the heart with saline, followed by $4 \%$ paraformaldehyde in $0.1 \mathrm{M}$ phosphate buffer at $\mathrm{pH}$ 7.4. Brains were removed, blocked coronally, and post-fixed for at least $24 \mathrm{hr}$ in the same fixative. Blocks of cortical tissue extending from the cruciate sulcus to the fork of the ansate and coronal sulci (Fig. 1) were embedded in paraffin and cut at $10 \mu \mathrm{m}$ thickness using a microtome. Sections were mounted on gelatin-subbed slides and stained to reveal Nissl substance, using cresyl violet. We specifically analyzed a region of area $3 b$ that contained the forepaw representation. The primary somatosensory cortex can be reliably identified by examining the relationships of gross structures, including the post cruciate dimple, the coronal and ansate sulci, and the anterior commissure in coronal sections (Juliano et al., 1996; McLaughlin et al., 1998). The cytoarchitecture of the selected region was examined starting rostrally using sections taken from the medial border of the cruciate sulcus and progressing caudally to the intersection of the ansate and coronal sulci. Five equally spaced coronal sections were chosen from within area $3 \mathrm{~b}$ of primary somatosensory cortex $(\sim 2 \mathrm{~mm}$ of tissue in the rostral to caudal plane) and used for detailed analysis. Area $3 b$ of ferret somatosensory cortex was identified according to the criteria described by McLaughlin et al. (1998). Briefly, area 3b includes a thick layer 4 with numerous densely packed granule cells and a relatively thin layer 5 with large pyramidal cells (compared with areas $3 \mathrm{a}$ and 4). Layers 2 and 3 contain smaller pyramidal cells, which are most prominent in layer 3 . Layer 2 also contains many smaller spherical cells, which are less densely packed than in layer 4. From the selected sections, a region of cortex lateral to the post cruciate dimple and medial to the coronal sulcus was digitally imaged extending from pia to white matter (Fig. 1). Using the Image Pro Plus program (Media Cybernetics, Silver Spring, MD), the thickness of cortex from pia to the bottom of layer 6 and the thickness of each individual layer was measured. A grid of boxes $500-\mu \mathrm{m}$-wide by $100-\mu \mathrm{m}$-deep starting from the top of layer 2 and extending to the bottom of layer 6 was created for each section; each box was assigned to a layer (Fig. 1). The Nissl-stained cells were counted in each box of normal and MAM-treated animals. Only Nissl-stained cells containing a single nucleolus within the nucleus were included for analysis. Small, presumptive glial cells and cells in which nucleoli were not present or could not be differentiated were excluded from analysis. We also measured the twodimensional surface area of each cell in which the nucleus contained a nucleolus. The data were imported into Microsoft (Seattle, WA) Excel, and $t$ tests were performed to compare between normal and E33 MAMtreated ferrets. Included in the analysis were comparisons between the overall thickness of cortex, the distribution of neuronal sizes through the depth of the cortex, the thickness of the individual cortical layers, the number of neurons within each box (as a measure of the packing density of neurons within a layer), and the average size of the individual cells per box.

DiI analysis. The sections were examined, and the labeled pathways were reconstructed using fluorescence microscopy and the data collection software Image Pro (Digital Solutions) and Neurolucida (MicroBrightField, Inc., Colchester, VT). All slices were examined on a fluorescent microscope outfitted with a CCD camera. Images were collected from each focal plane within an area of interest. These images were summed together to produce a flattened representation encompassing the entire $100 \mu \mathrm{m}$ thickness of a slice. We then imaged the same area of interest with the fluorescent bisbenzimide counterstain. From this image, we determined the boundaries of cortical layers based on cell morphology and nuclear densities. The borders of the cortical layers were superimposed on the flattened image of the thalamic afferents. With the limits of the cortical layers in place, we counted the number of labeled afferent fibers found within each identified laminar boundary. Because different brains were more or less densely labeled by the DiI, results were ex- pressed as a percent of the total labeled fibers identified within each block of cortex analyzed. These percentages were averaged between slices of the same brain to give a representative average value for each layer of a specific brain. It should be noted that a labeled fiber could be counted more than once if it was present in more than one layer. Each labeled fiber was carefully traced to its origin within a given section, and only fibers clearly originating from the white matter were included. This was to ensure that processes issuing from retrogradely labeled cells in the cortex were not mistaken for thalamic afferent fibers. If a fiber branched within a given layer, it was only counted once. A Mann-Whitney $U$ test was performed on the averages, comparing normal with E33 or E38 MAM-treated at each age. The E38 MAM-treated brains were excluded from statistical analysis at one age because there were fewer of these brains than either E33 MAM-treated or normal. The main intent of including the E38 treatment was to validate the specificity of the E33 MAM treatment.

In a representative set of animals, we assessed the volume of VB thalamus in normal and MAM-treated animals to verify that MAM injections on E33 did not alter its size and viability. To do this, the rostral border of the nucleus was delineated on horizontal sections, and landmarks observed in this plane were used as reference for definitive identification in coronal sections. Although a ferret atlas does not exist, many of the features of the ventrobasal nucleus are similar to those in cat, and the atlas of Berman and Jones (1982) was used as a guide. We included in our assessment nuclei identified in their nomenclature as ventrobasal arcuate, ventrobasal external, and basal ventromedial. We refer to them collectively as the VB. After identifying the rostral border on horizontal sections, we could easily identify VB in coronal sections stained with bisbenzimide. We delineated the borders of VB in the five most rostral sections (i.e., over $500 \mu \mathrm{m}$ ) of normal and E33 MAMtreated thalami. The boundary of this nucleus was outlined, and the volume was determined in normal and MAM-treated brains using ImagePro.

Electrophysiological recordings. Seven ferrets treated with MAM on E33 and 10 normal ferrets 6-8 months of age were used for electrophysiological recording. The preparation was as reported previously (McLaughlin et al., 1998). The ferrets were anesthetized with halothane (2-3\%); expired $\mathrm{CO}_{2}$ and body temperature were monitored and maintained within normal limits. Ophthalmic ointment was used in the eyes, heart rate was monitored, and 5\% dextrose in lactated Ringer's solution was delivered continuously intravenously. The animal's head was placed in a stereotaxic device, a craniotomy was performed over the right somatosensory cortex, and the dura was removed. The brain was covered with warm mineral oil and photographed. An enlarged photograph was then used to record the locations of individual electrode penetrations. Platinum iridium microelectrodes $(0.5-1 \mathrm{M} \Omega$ at $1 \mathrm{kHz})$ were used to record from small groups of cells. Cortical responses were amplified and monitored with an oscilloscope and audio monitor. Electrode penetrations were positioned either perpendicular to the cortical surface or angled along the medial wall of the coronal sulcus; penetration sites were separated by $200-500 \mu \mathrm{m}$. Cutaneous receptive fields (RFs) were determined by light stimulation of the skin with hand-held wooden probes. Deep pressure and/or joint manipulation were applied when sites did not respond to light cutaneous stimulation. The RFs were drawn on illustrations of ferret forepaw, forelimb, face and neck, and whole body. Von Frey hairs were used to determine the final boundaries of cutaneous receptive fields.

Behavioral observations. Although specific tests on the behavior of these animals were not conducted, they were observed on a daily basis until they reached 3 months of age. Until this age, they appeared to develop comparably with normal kits. Their behavior was not obviously unusual, and the physical appearance of the MAM-treated kits was similar to normal ferrets of their age. They had a good appetite and were playful.

\section{RESULTS}

Our analysis focussed on the somatosensory cortex. Although it was not possible to precisely identify somatosensory cortical areas by cytoarchitecture at all the ages studied, morphological landmarks were reliable indicators of the region (Juliano et al., 1996). The postcruciate dimple was visible at all ages that we used; the identified region corresponds to the hand region of area $3 \mathrm{~b}$ in the adult (Juliano et al., 1996; McLaughlin et al., 1999) (Fig. 1). For 
Figure 2. Digitized images of Nissl-stained coronal sections taken from normal $(A)$ and E33 MAM-treated $(B)$ neonatal ferrets at $\mathrm{P} 1$. In each section, the marginal zone and cortical plate (i.e., undifferentiated layers 2-4) are present; layers 5 and 6 can be identified but are not easily distinguishable. Although the MAM-treated cortex is slightly thinner, there are few observable differences between the two groups at this age. $m z$, Marginal zone; $c p$, cortical plate; $s p$, subplate. Scale bar, $250 \mu \mathrm{m}$.
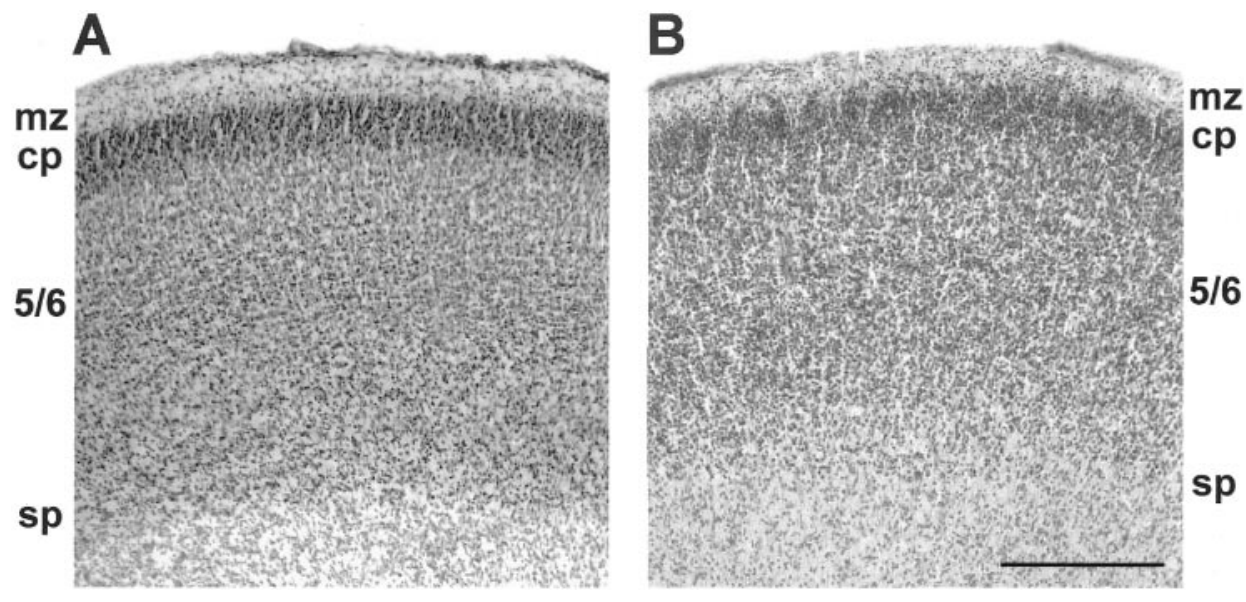

\section{Cortical Thickness}

P1

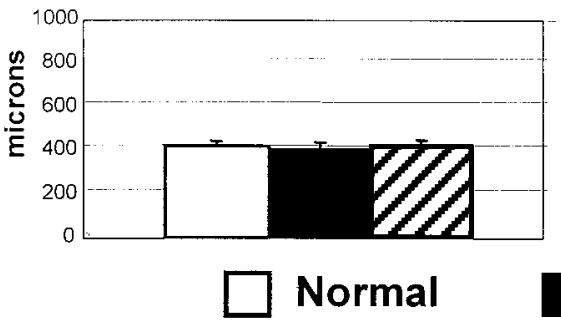

P7

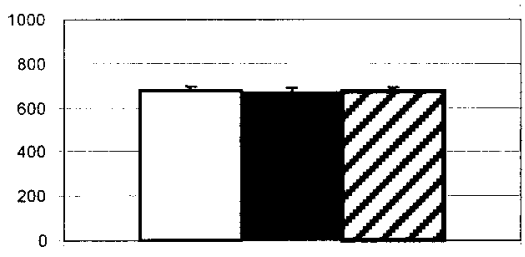

P14

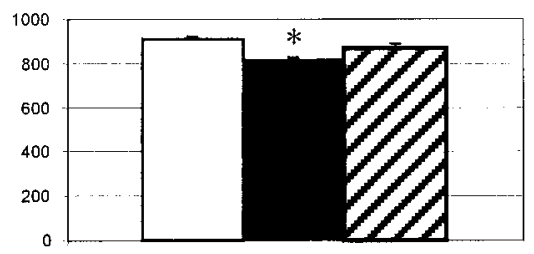

E33 MAM

Figure 3. Graph denoting the thickness of somatosensory cortex in normal and MAM-treated animals at different ages. At P1, cortical thickness is similar in all groups; the E33 MAM-treated cortex is slightly thinner, but this value is not significantly different from normal. At P7, the cortex is thicker, but there are still no significant differences between MAM-treated and normal. By P14, the E33 MAM-treated cortex is significantly thinner than normal $\left({ }^{*} p=0.004\right.$; two-tailed $t$ test $)$. SD is indicated with error bars.

our analysis of cytoarchitecture after injections of MAM, several developmental ages were assessed qualitatively, and quantitative analysis was conducted at 3 months, substantially pass the time when ferret somatosensory cortex reaches maturity (Juliano et al., 1996; Noctor et al., 1997). Thalamocortical connections after DiI injections into the thalamus were quantitatively analyzed at P1, P7, and P14. At P14 in ferret somatosensory cortex, the cortical layers are distinct, although they have not fully differentiated into mature morphology (Juliano et al., 1996). At ages older than P14, we found diffusion of DiI label unreliable.

\section{Cytoarchitectural analysis}

\section{Cytoarchitectural analysis of ferret somatosensory cortex at $P 0-P 1$}

At this age, neither the morphology nor cytoarchitecture of cortical regions are mature. At birth (P0-P1), Nissl-stained sections revealed very few differences between normal and MAMtreated brains. After MAM treatment, the marginal zone, a dense cortical plate (layers 2-4), differentiating layers 5-6, and the subplate are visible; this organization does not differ substantially from the appearance of the cytoarchitecture in normal neonatal ferrets (Fig. 2). After E33 MAM treatment, the neonatal cortex (P0-P1) was slightly thinner than that in normal neocortex or E38 MAM-treated cortex. The mean value of cortical thickness was $407 \mu \mathrm{m}$ in E33 MAM neonates compared with a mean of 414$\mu \mathrm{m}$-thick in normal neonates and $411-\mu \mathrm{m}$-thick in the E38 MAM-treated animals; these differences were not significant $(t$ test) (Fig. 3). This minimal difference is not surprising, because the normal laminar pattern is not yet established at birth and many cortical neurons are still migrating to cortex (Juliano et al., 1996; Noctor et al., 1997).

\section{Cytoarchitectural analysis of somatosensory cortex of 3-month-old ferrets}

Nissl-stained sections of primary somatosensory cortex were analyzed quantitatively using paraffin-embedded sections obtained from three normal ferrets and three E33 MAM-treated and two E38 MAM-treated ferrets at 3 months of age; at this time, the neocortex is cytoarchitecturally mature and the region of area $3 \mathrm{~b}$ containing the forepaw representation can be definitively identified (Juliano et al., 1996; McLaughlin et al., 1998). The remaining 3-month-old ferrets were assessed qualitatively.

Figure 4 illustrates Nissl-stained sections taken from an E33 MAM-treated cortex on the left and from normal ferret cortex on the right. In the section taken from normal ferret area $3 \mathrm{~b}$, cortical layers 1-6 are easily distinguished. In the E33 MAM-treated somatosensory cortex, layers $1,2,5$, and 6 were clearly present, but layers 3 and 4 are reduced in thickness and not as easily distinguished.

The histograms in Figure 5 demonstrate structural differences between MAM-treated and normal adult ferret somatosensory cortex. Figure $5 A$ demonstrates that E33 MAM-treated somatosensory cortex is significantly thinner than in normal animals, having a mean overall thickness of $1153 \mu \mathrm{m}$ versus $1519 \mu \mathrm{m}$ in the normal animals ( $p=0.03 ; t$ test). Also included in these charts are values obtained from the E38 MAM-treated animals. The thickness of the somatosensory cortex in the E38 MAM-treated 
E33 MAM

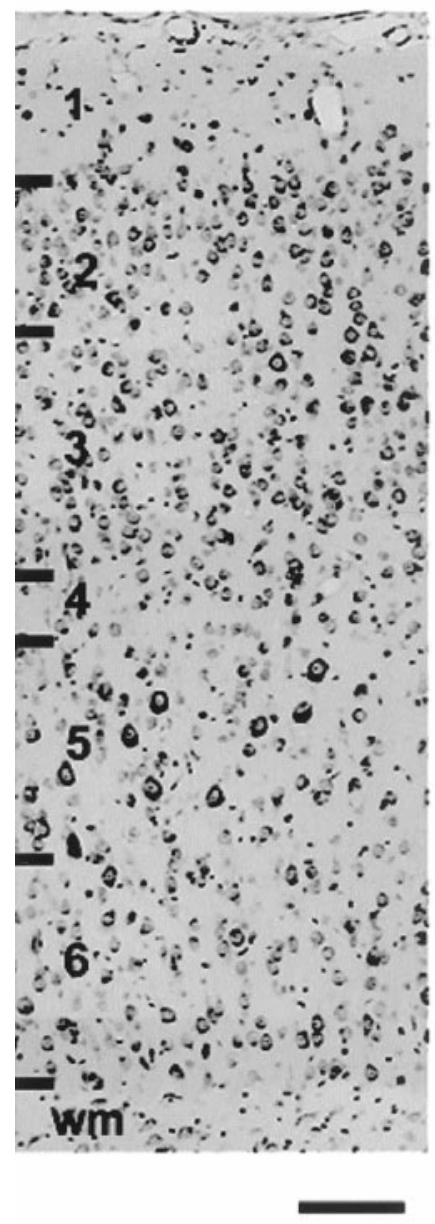

\section{Normal}

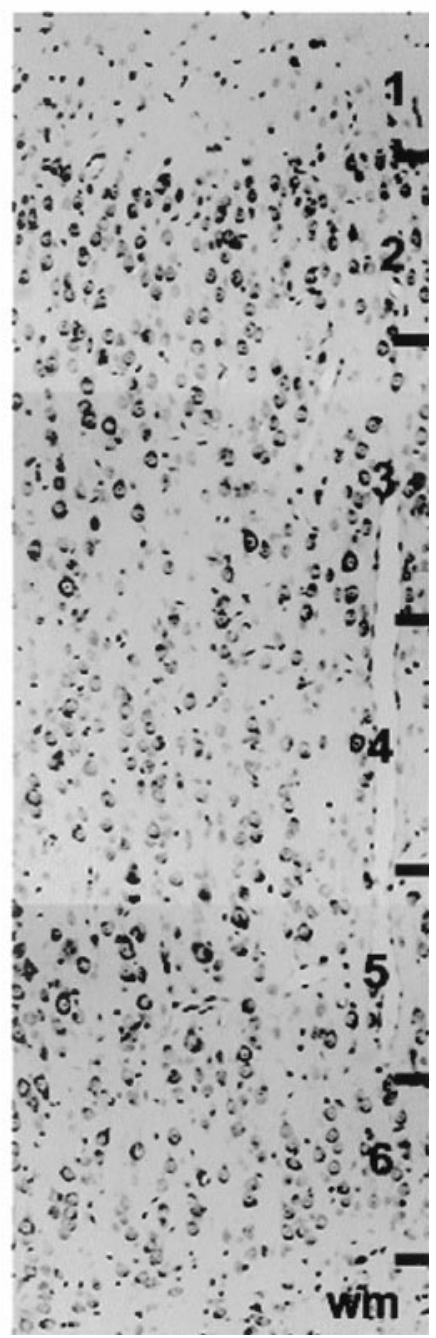

Figure 4. Digitized images of Nissl-stained sections of normal (right) and E33 MAM-treated (left) adult ferrets in the coronal plane. In the image taken from the normal animal, layers 1-6 can be clearly distinguished, whereas in the section taken from the E33 MAM-treated animal, the dimensions of layer 3 and 4 are greatly reduced. The remaining layers in the E33 MAM-treated animal $(1,2,5,6)$ are relatively normal in appearance. $w m$, White matter. Scale bar, $100 \mu \mathrm{m}$.

animals was $1438 \mu \mathrm{m}$ normal cortex. The thickness of layers 1,4 , 5, or 6 were not affected by E38 MAM treatment.

We counted the number of neurons in each box, as defined in Materials and Methods, to determine whether MAM injections influenced the packing density of the cells remaining in each layer of mature neocortex. Figure $5 C$ presents the mean number of neurons found per box in each layer for normal, E33 MAMtreated, and E38 MAM-treated ferrets. No differences in the overall density of neurons in layers 2, 3, 5, and 6 were observed between normal and E33 MAM-treated cortex. The density of neurons within layer 4 was reduced in the E33 MAM-treated adult ferrets compared with normal cortex; however, this difference was not significant ( $p<0.07 ; t$ test). The density of neurons within layers 3-6 did not differ between normal and E38 MAMtreated ferrets. The density of cells within layer 2, however, was reduced $23 \%$ compared with normal. We also measured the two-dimensional surface area of all cells containing nucleoli to determine whether E33 MAM or E38 MAM treatment altered the size of cells in the cortical layers. Cell surface area did not differ in any layer between the E33 MAM-treated and normal, or E38 MAM-treated and normal adult ferrets (Fig. 5D). MAM treatment, therefore, did not appear to alter the size of cells generated before or after administration of MAM.

\section{BrdU analysis}

Pregnant ferrets were injected with BrdU $3 \mathrm{~d}$ after MAM injection to ensure that cells continued to be born and migrate appropriately after MAM treatment. MAM injections did not prevent cells from being born and migrating to their proper position in the cerebral cortex. Figure 6 illustrates BrdU immunoreactivity in the somatosensory cortex of a mature animal that received MAM on E34 and a subsequent BrdU injection on E37. Also shown is BrdU immunoreactivity in a normal mature animal after BrdU injection on E33, which labels cells in layer 4. A discrete band of cells can be seen in each image. The BrdU-positive cells in the normal animal occupy a more inferior position, because the injection was earlier and the cells are more likely to reside in a deeper layer on this date. It can be seen that the BrdU-positive cells in the normal somatosensory cortex occupy a distinct laminar position, in layer 4 of somatosensory cortex; the labeled cells in the MAM-treated cortex also occupy a discrete laminar pattern appropriate for the date of embryonic development, in this case layer 3. It should also be noted that injections of BrdU at the time of MAM treatment result in little or no BrdU reactivity. The normal cortex is also thicker than the MAM-treated cortex because of the partial elimination of layers after MAM treatment.

\section{Dil injections \\ Thalamocortical projections in normal and MAM-treated brains at $P 1$}

At P1 in both the normal and MAM-treated cortex, the developing layers were poorly distinguished, as indicated above. The developing cortical plate consisted of the poorly defined layers 5 and 6 and the cell-dense, undifferentiated layers 2-4 (Fig. 2). In the normal ferret cortex at P1, labeled afferent fibers occur in lower layers 5 and 6 and subplate region. In E33 and E38 MAMtreated cortex, the majority of DiI-labeled thalamic fibers are also found within the lower layers of cortical plate (Fig. 7). Occasional labeled fibers appear within the undifferentiated cortical plate in all treatment groups, although they usually do not end there but ultimately terminate in layer 1 . Many of the labeled afferent thalamic fibers travel tangentially in the white matter and immature lower cortical layers, as has been reported by a number of other groups; eventually they turn to enter the cortical plate with a vertical approach (Catalano et al., 1991; Agmon et al., 1993; Juliano et al., 1996). In the quantitative analysis, the number of fibers counted in each layer was expressed as a percent of the total number of labeled fibers. The mean number of fibers counted in each layer for all animals in all conditions are shown in Table 2. An example of some of the factors contributing to this analysis is illustrated in Figure 8. In this figure, examples of the fibers counted for analysis are highlighted in red. By focusing through the thickness of the section, only fibers that were traced to originate in the white matter were included in the tally. Examples of fibers excluded for analysis are shown in green or blue. These included processes that were attached to cortical cells or those for which we could not clearly observe an origin. At P1, the following layers were identified for analysis: layer 1 , the dense, undifferen- 
A

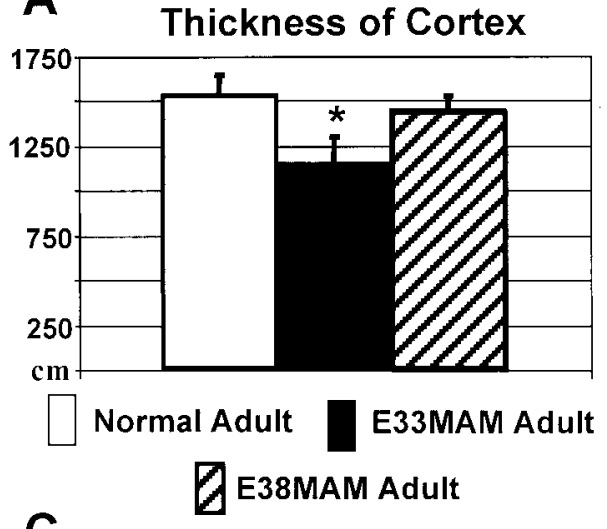

C

Cell Packing Density Per Layer

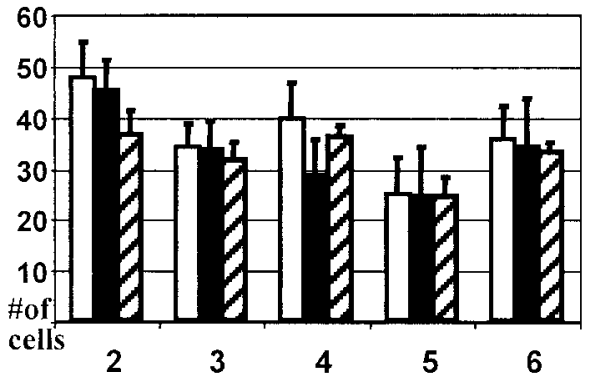

B

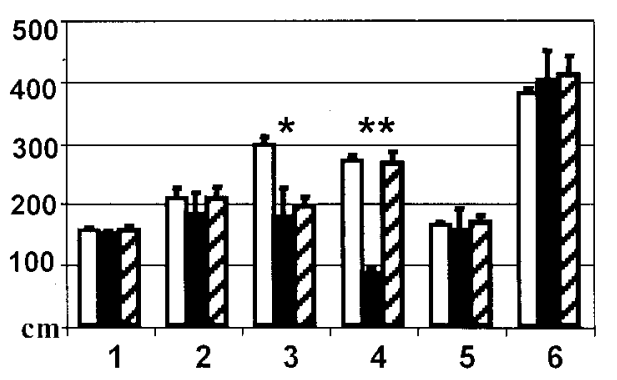

D

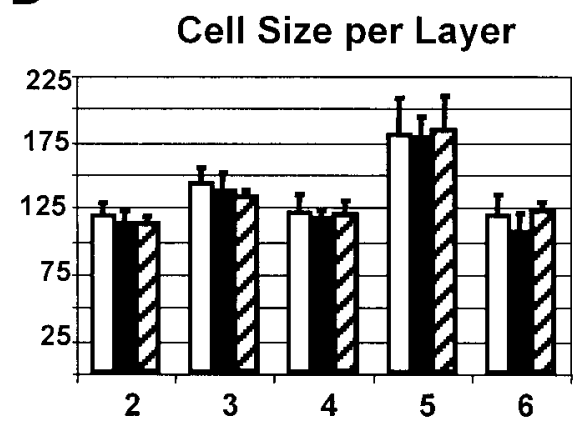

Figure 5. Histograms comparing specific features of cortical architecture in normal (white bars), E33 MAM-treated (black bars), and E38 MAM-treated (striped bars) adult ferrets. The data represent average values obtained from three animals in the normal and E33 MAM-treated groups and two animals in the E38 MAM-treated group. Because of the lower number of E38 MAMtreated animals, they were not included in statistical analysis. $A$, Measurements of overall cortical thickness in the adult animals indicate that E33 MAM cortex is significantly thinner than normal cortex $\left({ }^{*} p<\right.$ $0.03)$; the E38 MAM-treated cortex is slightly thinner than normal cortex. $B$, Measurements of individual cortical layers indicate that layer $3\left({ }^{*} p<0.03\right)$ and layer 4 $\left({ }^{* *} p<0.01\right)$ were responsible for the overall difference in cortical thickness, because they were significantly thinner in the E33-MAMtreated animals, whereas the other layers were similar in thickness. Layer 3 was also thinner in the E38 MAM-treated animals. $C$, Measurements of overall cell density did not reveal significant differences between normal and MAM-treated cortex. Cell density was slightly lower in layer 4 , but this difference was not significant ( $p=0.07 ; t$ test). \# of cells on the $y$-axis refers to the mean number of cells per bins assigned to a given layer. $D$, Cell size, as determined by areal measurements, did not differ in any layer when comparing measurements between MAM-treated and normal ferrets. The numbers on the $x$-axis of $B-D$ represent cortical layers. Values are expressed in square micrometers. Error bars indicate the SD. tiated cortical plate $(C P)$, layer 5, and layer 6 (Fig. 7). The quantitative analysis revealed no significant differences between groups in the distribution of total fibers within the analyzed layers (Mann-Whitney $U$ test) (Fig. 9).
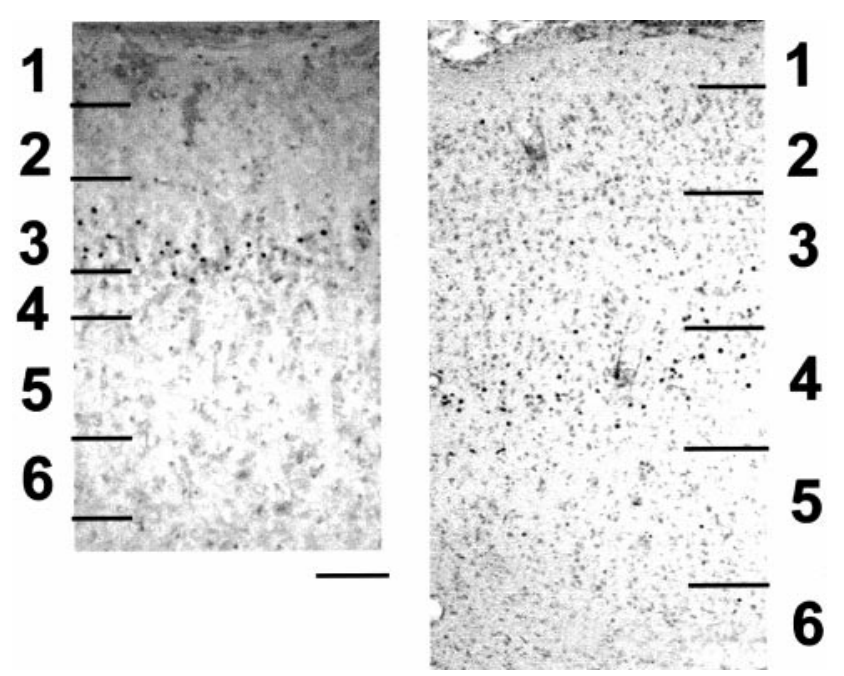

Figure 6. On the left is a photomicrograph of BrdU immunoreactivity in mature ferret cortex (P44) that received BrdU 3 d after E34 MAM treatment. The BrdU-positive cells are located in an appropriate layer of somatosensory cortex (i.e., layer 3), indicating that neurogenesis and migration resumed after the E33 MAM treatment. Shown on the right is an example of BrdU reactivity in a normal adult ferret that was injected with BrdU on E33, which normally labels layer 4. Scale bar, $100 \mu \mathrm{m}$.

\section{Thalamic projections in normal and MAM-treated brains at P7}

At P7, the cytoarchitecture of somatosensory cortex is similar to that seen at P1 (Juliano et al., 1996). The cortex is thicker, however, and layers 5 and 6 are more easily distinguished; otherwise, the laminar features are comparable with those observed on P1. There were no significant differences in thickness observed between the different treatment groups (i.e., normal or MAMtreated) (Fig. 3). In thalamocortical development, subtle differences begin to emerge between normal, E33, and E38 MAMtreated brains by P7 (Figs. 7, 9).

Quantitative analysis of percentages of the total numbers of labeled fibers finds that, in all groups, the greatest percentage of fibers are found in the deeper layers (Fig. 9). These afferent fibers are most likely traveling through to terminate in the more superficial layers: layer 4 in the normal and E38 MAM-treated animals. In the E33 MAM-treated brains, significantly more fibers are distributed in the cortical plate $(p=0.048)$ and layer $1(p=$ 0.012 ) compared with those distributions in normal animals. The E38 MAM-treated brains also had a high number of afferent fibers in the cortical plate.

\section{Thalamic projections in normal and MAM-treated brains at $P 14$}

The most obvious differences between normal and E33 MAMtreated brains occur at P14. At this age, the normal laminar architecture is more distinct and each cortical layer can be identified, although they have not attained complete maturity (Juliano 

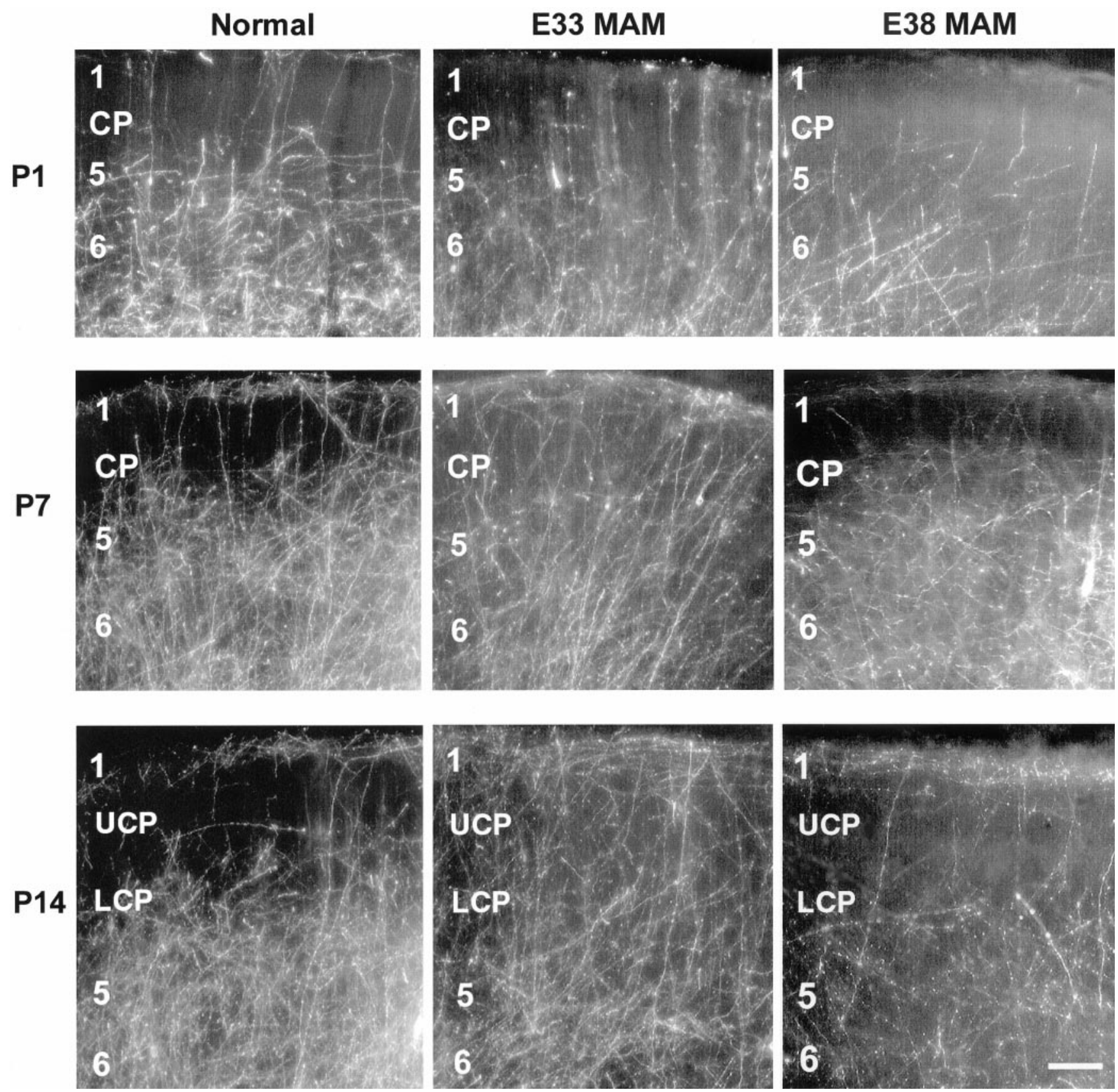

Figure 7. DiI label in the somatosensory cortex of normal and MAM-treated animals after injections in VB thalamus. At P1 (top row), labeled fibers can be seen primarily in the deeper aspects of the cortex (immature layers 5 and 6). At P7 (middle row), distinctions between the thalamic terminations after MAM treatment can be observed, with more fibers ending toward the upper portion of the cortex after E33 MAM treatment compared with either normal or E38 MAM-treated cortex. At P14 (bottom row), the thalamic terminations are almost equally distributed through all cortical layers in the E33 MAM-treated brains. This is in contrast to the label observed in the normal and E38 MAM-treated animals, which contain thalamic terminations more focussed toward the central and lower layers. Scale bar, $100 \mu \mathrm{m}$. $C P$, Cortical plate; $L C P$, lower cortical plate; $U C P$, upper cortical plate.

et al., 1996). In the MAM-treated animals, the specific layer targeted is diminished in dimension. For this analysis, we identified the following laminar distinctions: layer 1, upper cortical plate (presumptive layers 2-3), lower cortical plate (presumptive layer 4), and layers 5 and 6 . The somatosensory cortex is significantly thinner in the E33 MAM-treated animals compared with normal (two-tailed $t$ test; $p=0.004$ ) (Fig. 3). Organization of thalamic afferents is strikingly different between E33 MAMtreated and control cortices (Figs. 7, 9). In the normal somato- sensory cortex, the greatest percentage of DiI-labeled thalamic afferent fibers distribute in the lower layers and in the lower cortical plate; very few fibers extend beyond this point. Many labeled thalamic fibers projecting into the cortex in the E33 MAM-treated animals fail to terminate within the lower layers or lower cortical plate and continue into upper layers, including layer 1. The distribution patterns in the E33 MAM-treated brains show almost equal percentages of fibers distributed across cortical layers compared with the bulk of fibers in the lower cortical plate 
Table 2. Mean number of fibers in somatosensory cortex area $3 \mathrm{~b}$ labeled with DiI after injection in ventrobasal thalamus and counted in different cortical layers for all animals

\begin{tabular}{lllll} 
& \multicolumn{4}{l}{ Cortical layers at P1 } \\
\cline { 2 - 5 } & 1 & CP & 5 & 6 \\
\hline Normal & 11.1 & 18.3 & 24.4 & 28.7 \\
E33 MAM & 10.4 & 13.8 & 23.1 & 33.4 \\
E38 MAM & 8.3 & 17.7 & 38.7 & 39.38
\end{tabular}

\begin{tabular}{lrlll} 
& \multicolumn{5}{c}{ Cortical layers at P7 } \\
\cline { 2 - 5 } & \multicolumn{1}{c}{1} & CP & 5 & 6 \\
\hline Normal & 7.1 & 11.4 & 37.6 & 43.9 \\
E33 MAM & 19.4 & 15.6 & 28.9 & 36.0 \\
E38 MAM & 8.9 & 20.1 & 29.6 & 31.8 \\
\hline
\end{tabular}

\begin{tabular}{|c|c|c|c|c|c|}
\hline & \multicolumn{4}{|c|}{ Cortical layers at P14 } & \multirow[b]{2}{*}{6} \\
\hline & 1 & $\mathrm{UCP}$ & LCP & 5 & \\
\hline Normal & 6.9 & 10.4 & 23.8 & 31.2 & 27.4 \\
\hline E33 MAM & 17.9 & 15.7 & 17.9 & 20.7 & 21.9 \\
\hline E38 MAM & 8.2 & 10.5 & 23.5 & 29.1 & 28.7 \\
\hline
\end{tabular}

See Results for details. CP, Cortical plate; UCP, upper cortical plate; LCP, lower cortical plate.

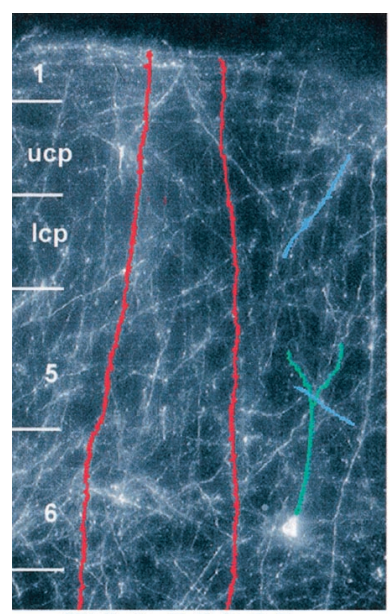

E33 MAM

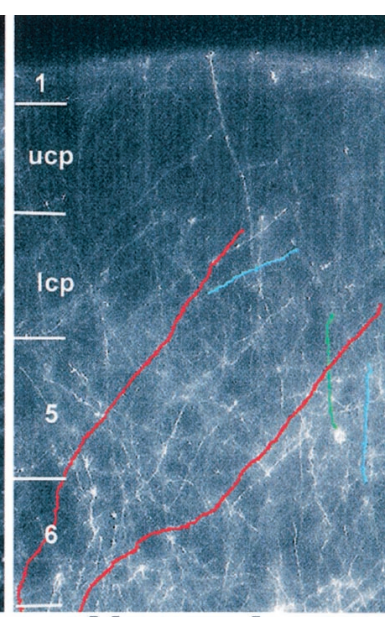

Normal
Figure 8. Examples of DiI label after injection into the ventrobasal thalamus of normal and E33 MAM-treated brains at P14. Highlighted in color (red) are fibers that are clearly identified as originating from the white matter and followed to their termination by focusing through the thickness of the section. Also indicated are examples of fibers that are excluded from the tally, either because their origin could not be clearly identified (blue) or because they were attached to a retrogradely labeled cell (green). The red fibers in the E33 MAM-treated section are counted five times because they are present in five layers $(1,5,6, l c p, u c p)$, whereas the red fibers in the normal section are counted three times because they are present in three layers $(5,6, l c p)$. $u c p$, Upper cortical plate; $l c p$, lower cortical plate.

and layer 5 in normal and E38 MAM-treated cortex. These data achieve statistical significance (Fig. 9).

\section{Measurements of ventrobasal thalamus}

To determine that MAM treatment timed to interfere with layer 4 development did not dramatically alter the nature of the thal- amus projecting to the somatosensory region, we measured the volume of VB in normal and E33 MAM-treated animals. This analysis determined that there were no significant differences in the size of $\mathrm{VB}$ at any of the ages examined: P1, P7, and P14 (Table 3). We realize that these data do not provide information about the cellular composition of the normal or MAM-treated thalami but provide a global view of the overall size of the ventrobasal thalamic nuclei, which does not change after E33 MAM treatment.

\section{Electrophysiological recordings}

Recordings were conducted in the somatosensory cortex of normal or E33 MAM-treated ferrets at a depth of 500-800 $\mu \mathrm{m}$, a level presumed to correspond to the central layers. In addition to demonstrating the functional integrity of thalamic projections onto cortex, despite their disarray, we observed that the topographic representation of the body is almost normal in E33 MAM-treated somatosensory cortex. The cutaneous representation in the crown of the posterior sigmoid gyrus is of normal size and contains an orderly arrangement of cells with a typical systematic shift in receptive fields (Fig. 10). Proximal portions of the arm were represented medial to the forepaw, and the usual shift within the forepaw representation of digit 5 most medially to digit 1 most laterally was evident. The sizes of the receptive fields recorded in the somatosensory cortex were also normal, as was the distribution of submodality properties, which displayed typical shifts across presumptive cytoarchitectonic fields. In both E33 MAM-treated and normal animals, injections of fluorescently labeled dextrans (Fluororuby) were made in selected penetrations to verify recording depth and cytoarchitecture in all animals.

\section{Summary and use of $M A M$}

Our current experiments indicate that treatment with MAM disrupts the generation of specific populations of neocortical cells, after which corticogenesis resumes. The thickness of layer 4 was reduced by nearly $70 \%$ and layer 3 was reduced by $40 \%$ after E33 MAM treatment, yet somatosensory cortex retained much of its characteristic appearance. Furthermore, the neurons generated after MAM treatment were appropriate in morphology, size, and density. Interference with the development of layers 3 and 4 using appropriately timed injections of MAM also results in the altered distribution of thalamocortical projections. At P14 in ferrets, projections from VB are almost uniformly distributed among all neocortical layers when layer 4 is diminished, rather than being focussed in the central region. MAM injections timed to interfere with the development of layer 2-3 do not produce the same alteration of thalamic terminations and result in a pattern of projections similar to those observed in normal animals. Despite the change in thalamic projection pattern, the cortical responses to somatic stimulation in E33 MAM-treated cortex were similar in topographic and submodality distribution, as well as receptive field size.

\section{DISCUSSION}

\section{Specificity of MAM treatment}

MAM methylates the $7^{\prime}$ position of guanine in DNA and RNA, forming 7-methyl-guanine. This results in decreased cell division, which recovers within $24 \mathrm{hr}$ (Matsumoto and Higa, 1966; Zedeck et al., 1970; Evans and Jenkins, 1976). The short-term effect of MAM on cell division prevents the generation of specific populations of neurons through a single administration on an appropriate gestational date. This is supported by Cattaneo et al. 


\section{P1}

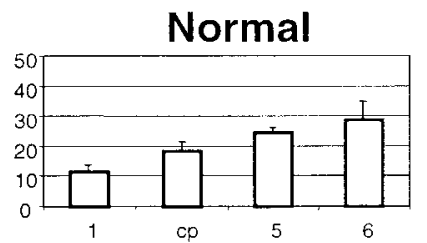

\section{Normal}
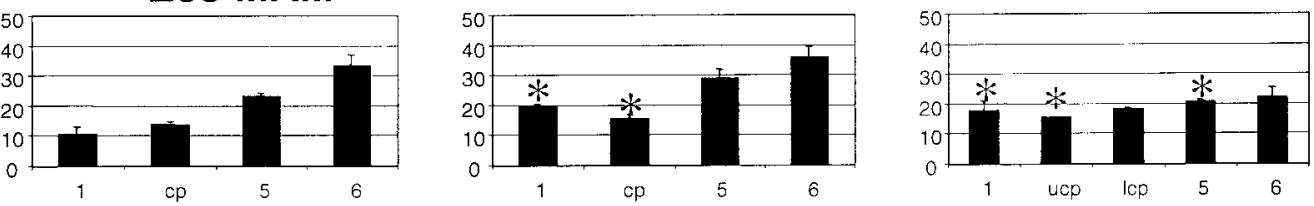

P14

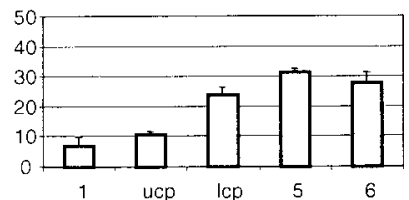

\section{E38 MAM}

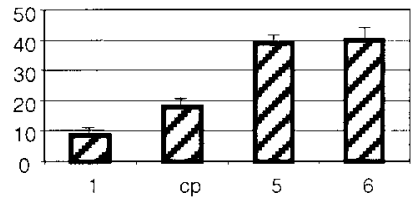

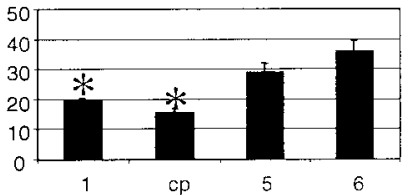

P7
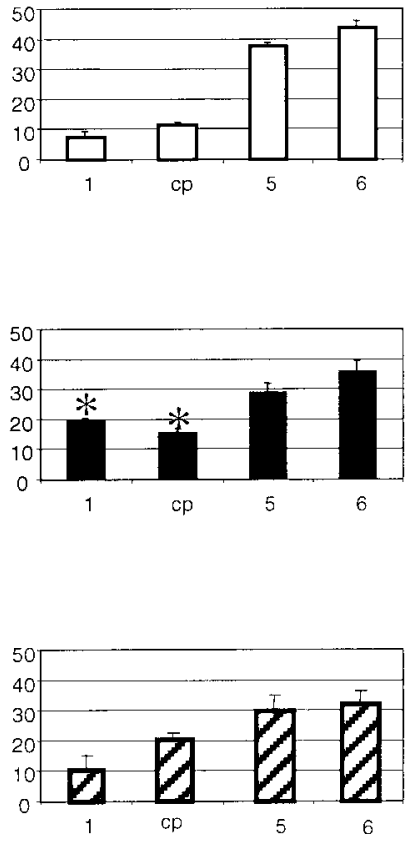

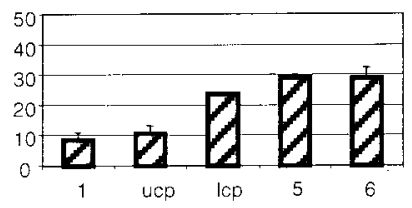

Figure 9. Left column, Graphs indicating the distribution of thalamocortical fibers in the cerebral cortex at P1 in normal, E33, and E38 MAM-treated somatosensory cortex. At this age, the following layers could be distinguished: layer 1 , the undifferentiated cortical plate ( $c p$ ), and layers 5 and 6 . The values in each layer (indicated on the $x$-axis) are expressed as a percentage of the total fibers (indicated on the $y$-axis) found in all cortical layers. There were no significant differences between any of the groups compared with normal (Mann-Whitney $U$ test). Middle column, Graphs indicating the distribution of thalamocortical fibers in the cerebral cortex at P7 in normal, E33, and E38 MAM-treated somatosensory cortex. At this age, the following layers are distinguished: layer 1 , the undifferentiated cortical plate $(c p)$, and layers 5 and 6 . The values in each layer ( $x$-axis) are expressed as a percentage of the total fibers found in all cortical layers $(y$-axis). The number of thalamic fibers in layer $1(p=0.012)$ and the cortical plate $(p=0.048)$ were significantly greater in the E33 MAM-treated animals compared with normal (asterisks). Right column, Graphs depicting the distribution of thalamocortical fibers in the cerebral cortex at P14 in normal, E33, and E38 MAM-treated somatosensory cortex. At this age, the following layers are distinguished: layer 1, the upper part of the cortical plate (ucp; presumptive layers 2-3), the lower part of the cortical plate (lcp; presumptive layer 4), and layers 5 and 6 . The values in each layer are expressed as a percentage of the total fibers found in all cortical layers. The amount of thalamic fibers in layer $1(p=0.05)$, the upper cortical plate $(p=0.021)$, and layer $5(p=0.009)$ were significantly greater in the E33 MAM-treated animals compared with normal (asterisk). The error bars indicate SD.

\begin{tabular}{lll}
\hline \multicolumn{2}{l}{ Table 3. Volume of anterior $\mathbf{5 0 0} \boldsymbol{\mu m}$ of ventral basal thalamus in $\mathbf{~ m m}^{\mathbf{3}}$} \\
& Normal & E33 MAM \\
\hline P1 & 0.1267 & 0.1277 \\
P7 & 0.1754 & 0.1805 \\
P14 & 0.2049 & 0.1981
\end{tabular}

Values represent means obtained from three animals at each age and treatment. Differences between normal and treatment at each age were not statistically significant.

(1995) reporting that MAM transiently blocks neuroepithelial cell proliferation but does not interfere with cell survival. Cattaneo et al. (1995) also reported that MAM halts cell division in neuronal cell cultures but does not affect morphology or generation of glial cell cultures. We demonstrated previously that neurogenesis resumes within $24 \mathrm{hr}$ after MAM injection in ferrets, together with the almost normal birth of cells and migration of neurons (Noctor et al., 1999).

Delivery of MAM results in discrete and specific disruption of either layers 3 and 4 (E33 delivery) or layers 2-3 (E38 delivery) in ferret somatosensory cortex. The overall size of the most significant thalamic nucleus projecting to the somatosensory cortex, the ventrobasal nucleus, is not altered. Although features of thalamic organization may be altered by E33 or E38 MAM treatment, ancillary data do not support this prospect. The cells populating VB are born primarily before MAM injection (Bayer and Altman, 1991; Johnson and Casagrande, 1993). Woo and Finlay (1997) determined that the lateral geniculate nucleus contained a normal number of cells in MAM-treated hamsters having selective layer 4 lesions of visual cortex. The effects of MAM are highly specific to the date of the injection, implying that MAM acts through a narrow window of time leading to interruption of specific cell populations.

Our experiments did not completely eliminate layer 4 and also resulted in diminution of layer 3. This is not unexpected, because each layer is born over a period of several days (Noctor et al., 1997). We also cannot guarantee that all the cells in the somatosensory cortex reside in their proper sites. There may be cells formerly destined for layer 4, which now lie in layer 2 or 3. Two arguments imply the resulting laminar distribution of cortical cells is not grossly disordered after the MAM treatments. First, the cellular morphologies in MAM-treated animals, which typify given layers, remain comparable with those in normal animals. Second, BrdU injection, 3 d after an E33 or E34 MAM injection, produces BrdU-positive cells in a position appropriate for their date of birth in normal animals. These findings support the idea that cells born after the MAM injection migrate to positions 

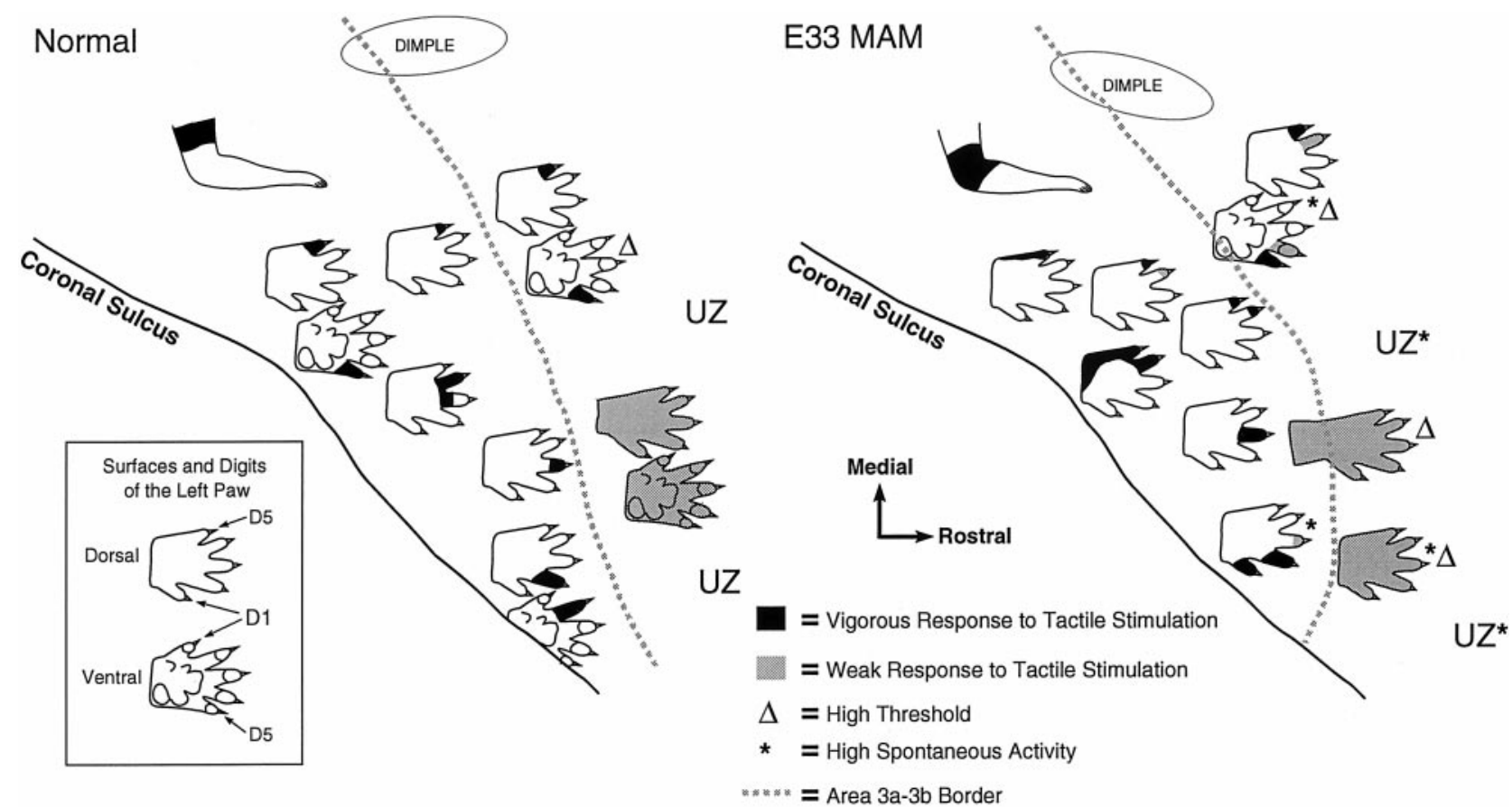

Figure 10. These are examples of the topographic distribution of the receptive fields recorded in a representative normal (left) and E33 MAM-treated (right) cortex. The receptive field organization of the forepaw in the MAM-treated cortex was similar to that in the normal cortex. Proximal fields were found medially in normal and MAM-treated brains, and the typical medial to lateral progression of digits 5 through 1 was also observed in both normal and MAM-treated somatosensory cortex. Responses to deep stimuli and/or reduced responsivity to light cutaneous stimuli were found rostrally, representing the progression into area 3a. Receptive field sizes were also similar for both normal and E33 MAM-treated cortex. These distributions are typical of 10 normal and seven E33 MAM-treated animals. UZ, Unresponsive zone; DIMPLE, location of the postcruciate dimple.

appropriate to their normal date of birth and do not redistribute in abnormal loci.

\section{MAM effects on layer 4}

Woo and Finlay (1996) determined that, despite almost complete destruction of layer 4 in the visual cortex of the hamster, the lateral geniculate nucleus projects a bilaminar pattern on the visual cortex. Jones et al. (1982) and Yurkewicz et al. (1984) also found a bilaminar distribution of thalamic projections in the somatomotor cortex of the rat after MAM-induced disruption of layers 2-4. Jones and colleagues suggested that the thalamic terminals preferred sites that normally provided an attraction, whereas Woo and Finlay concluded that it was not necessary for the thalamic projections to terminate on specific neurons but could distribute to almost any available cell.

In our experiment, the thalamic projections did not terminate in a distinct laminar pattern but distributed almost equally through all cortical layers. It is not clear why the cells remaining in layer 4 did not receive a disproportionate number of thalamic terminations but indicates that components other than those belonging to layer 4 can serve as targets. The previous studies also suggest that other sites may serve as targets, because layer 4 was primarily missing, but observed thalamic terminations occurring in close proximity to their normal terminal site. Because the ferret has a more protracted gestation than rats or hamsters, MAM treatment may more specifically and differentially eliminate a cortical layer depending on the time of injection.

\section{Neocortical influence on thalamic termination}

Thalamic fibers operate under a degree of autonomy as they grow toward the neocortex, suggesting that initial axonal outgrowth may occur without cortical cues (Molnar et al., 1999). Despite this initial independence, many studies support the idea that the neocortex exerts a tropic effect on axons leaving the thalamus. In vitro studies evaluating conditions of axonal growth report that pieces of thalamus grown independently emit fewer fibers than cultured slices grown in the proximity of cortical pieces (Molnar and Blakemore, 1999). Also supporting the idea that the cortex exerts a tropic effect on the thalamus are observations that specific molecules intrinsic to cortex attract thalamic axons (Barbe and Levitt, 1992; Mann et al., 1998).

Whether or not details of the topographic arrangements and thalamic arborization refine under neocortical control are subjects of intense scrutiny. Many studies support the idea that details of topographic projections and thalamic arborization are strongly influenced by features of the neocortex. The presence of intrinsic neocortical activity is a likely candidate for influencing thalamic refinement. Dating over several decades, studies involving the visual system, beginning with the work of Hubel et al., find that silencing or reducing activity in the cortex by limiting afferent input results in aberrant thalamic terminations, suggesting that the intrinsic activity of the cortex strongly influences the detail of thalamic termination (Hubel et al., 1977; Stryker and Harris, 1986; Antonini and Stryker, 1996; Katz and Shatz, 1996). In our study, although activity is not eliminated, it is most likely altered by the reduction of layers 3 and 4 , which could contribute to the disordered distribution of thalamic fibers.

\section{What is the nature of layer $\mathbf{4}$ influence?}

There are two obvious functions that layer 4 cells might conduct during thalamocortical growth: attract ingrowing afferents and/or instruct afferent fibers to stop. If layer 4 cells attract growing thalamic axons, the thalamocortical trajectory should be dis- 
turbed in the relative absence of layer 4 . Our observations demonstrate, however, that thalamic afferents project directly to the appropriate region of cortex in E33 MAM-treated animals. The electrophysiological studies find that topography is preserved in ferrets receiving MAM injections interfering with layer 4 production, indicating preservation of positional relationships in thalamic projections. Although cortical influence may be important for thalamic attraction, layer 4 is not required for this process.

Nevertheless, many authors conclude that layer 4 provides important cues instructing thalamic afferent termination and suggest that thalamic afferents will not grow into the neocortex until a specific level of maturity is achieved, which presumably coincides with the arrival of layer 4 (Gotz et al., 1992; Bolz, 1994; O'Leary et al., 1994; Molnar and Blakemore, 1995, 1999; Molnar et al., 1999). In vitro studies of cortical slices cultured with pieces of thalamus report that axons leaving the thalamus terminate in layer 4, regardless of whether the thalamic piece is juxtaposed to the pial or subcortical surface (Gotz et al., 1992; Yamamoto et al., 1997; Molnar and Blakemore, 1999). Other studies of organotypic cocultures report that growing thalamic axons appear to recognize layer 4, in which they stop, branch, and display growth cone collapse (Yamamoto et al., 1997). Even in a deficiency of these explicit signals in the E33 MAM-treated animals, afferent fibers eventually stop and branch, suggesting that without specific layer 4 cues, arbors form. This implies that other mechanisms participate in thalamic arbor formation and may include features that play a role in producing topographic precision, such as the activity of cortical cells, whether extrinsically or intrinsically derived. Again, our studies do not rule out the possibility that layer 4 cells may be displaced after MAM treatment and influence thalamic arborization while in inappropriate positions.

\section{How does diminution of layer 4 affect function?}

Overall topography is preserved within the somatosensory cortex of E33 MAM-treated animals; receptive field size and submodality shifts also seem normal. In addition, although not tested behaviorally, the ferrets appear typical in their activities. A centrally located band of layer 4 cells appear not to be highly important in determining the cortical body map, because topographic relations are well preserved in ferrets with a severely diminished layer 4. This indicates that the information carried on thalamocortical afferent fibers are an important factor in determining the body map. Despite the normality of body plan and receptive field size, preliminary results from electrophysiological recordings in the MAM-treated animals find altered laminar responses using current source density profiles. In the E33 MAMtreated somatosensory cortex, rather than initial sinks of activity observed in layer 4 , the incoming activity was equally distributed throughout all layers, mimicking the distribution of thalamic afferents (McLaughlin and Juliano, 1999). Additional recordings indicate that frequency after capabilities in E33 MAM-treated somatosensory cortex are impaired compared with normal animals (McLaughlin and Juliano, 1999). These observations imply that cortical responses in animals lacking most of layer 4 are modified to reflect the altered distribution of thalamic fibers. Our findings also suggest that qualities present in layer 4 are important to properly convey specific features of stimuli for further cortical processing, but that the body map can be accurately represented without an intact layer 4 .

\section{REFERENCES}

Agmon A, Yang LT, O’Dowd DK, Jones EG (1993) Organized growth of thalamocortical axons from the deep tier of terminations into layer IV of developing mouse barrel cortex. J Neurosci 13:5365-5382.

Antonini A, Stryker MP (1996) Plasticity of geniculocortical afferents following brief or prolonged monocular occlusion in the cat. J Comp Neurol 369:64-82.

Barbe MF, Levitt P (1992) Attraction of specific thalamic input by cerebral grafts depends on the molecular identity of the implant. Proc Natl Acad Sci USA 89:3706-3710.

Bayer SA, Altman J (1991) Neocortical development. New York: Raven. Berman A, Jones E (1982) The thalamus and basal telencephalon of the cat. Madison, WI: The Univeristy of Wisconsin.

Bicknese AR, Sheppard AM, O'Leary DDM, Pearlman AL (1994) Thalamocortical axons preferentially extend along a chondroitin sulfate proteoglycan-enriched pathway coincident with the neocortical subplate and distinct from the efferent path. J Neurosci 14:3500-3510.

Bolz J (1994) Cortical circuitry in a dish. Curr Opin Biol 4:545-549.

Catalano SM, Robertson RT, Killackey HP (1991) Early ingrowth of thalamocortical afferents to the neocortex of the prenatal rat. Proc Natl Acad Sci USA 88:2999-3003.

Cattaneo E, Reinach B, Caputi A, Cattabeni F, Di Luca M (1995) Selective in vitro blockade of neuroepithelial cells proliferation by methylazoxymethanol, a molecule capable of inducing long lasting functional impairments. J Neurosci Res 41:640-647.

Evans LA, Jenkins EC (1976) PHA response and methylazoxy methanol acetate. Chem Biol Interact 14:135-140.

Gotz M, Novak N, Bastmeyer M, Bolz J (1992) Membrane bound molecules in rat cerebral cortex regulate thalamic innervation. Development 116:507-519.

Hubel DH, Wiesel TN, LeVay S (1977) Plasticity of ocular dominance columns in monkey striate cortex. Philos Trans R Soc Lond B Biol Sci 278:377-409.

Hubener M, Gotz M, Klostermann S, Bolz J (1995) Guidance of thalamocortical axons by growth-promoting molecules in developing rat cerebral cortex. Eur J Neurol 7:1963-1972.

Jackson CA, Peduzzi JD, Hickey TL (1989) Visual cortex development in the ferret. I. Genesis and migration of visual cortical neurons. J Neurosci 9:1242-1253.

Johnston JK, Casagrande VA (1993) Prenatal development of axon outgrowth and connectivity in the ferret visual system. Vis Neurosci 10:117-130

Jones EG, Valentino KL, Fleshman Jr JW (1982) Adjustment of connectivity in rat neocortex after prenatal destruction of precursor cells of layers ii-iv. Brain Res 254:425-431.

Juliano SL, Palmer SL, Sonty RV, Noctor SC, Hill GF (1996) Development of local connections in ferret somatosensory cortex. J Comp Neurol 374:259-277.

Katz LC, Shatz CJ (1996) Synaptic activity and the construction of cortical circuits. Science 274:1133-1138.

Mann F, Zhukareva V, Pimenta A, Levitt P, Bolz J (1998) Membraneassociated molecules guide limbic and nonlimbic thalamocortical projections. J Neurosci 18:9409-9419.

Matsumoto H, Higa HH (1966) Studies in methylazoxy methanol, the aglycone of cycasin: methylation of nucleic acids in vitro. Biochem $\mathbf{J}$ 98:20C-22C

McLaughlin DF, Juliano SL (1999) Absence of layer 4 leads to impairment of laminar-specific response entrainment in ferret somatosensory cortex. Soc Neurosci Abstr 25.

McLaughlin DF, Sonty RV, Juliano SL (1998) Organization of the forepaw representation in ferret somatosensory cortex. Somatosens Mot Res 15:253-268.

Miller B, Sheppard AM, Bicknese AR, Pearlman AL (1995) Chondroitin sulfate proteoglycans in the developing cerebral cortex: the distribution of neurocans distinguishes forming afferent and efferent axonal pathways. J Comp Neurol 355:615-628.

Miller MW, Nowakowski RS (1988) Use of bromodeoxyuridineimmunohistochemistry to examine the proliferation, migration and time of origin of cells in the central nervous system. Brain Res 457:44-52.

Molnar Z, Blakemore C (1995) Guidance of thalamocortical innervation. In: Development of the cerebral cortex (Bock G, Cardew G, ed), pp 127-139. Chichester, UK: Wiley.

Molnar Z, Blakemore C (1999) Development of signals influencing the growth and termination of thalamocortical axons in organotypic culture. Exp Neurol 156:363-393.

Molnar Z, Adams R, Blakemore C (1998) Mechanisms underlying the early establishment of thalamocortical connections in the rat. J Neurosci 18:5723-5745.

Molnar Z, Higashi S, Adams R, Toyama K (1999) Earliest interactions between thalamus and cortex. In: The barrel cortex (Kossut M, ed). London: Graham, in press.

Noctor SC, Scholnicoff NJ, Juliano SL (1997) Histogenesis of ferret somatosensory cortex. J Comp Neurol 387:179-193. 
Noctor SC, Palmer SL, Hasling T, Juliano SL (1999) Interference with the development of early generated neocortex results in disruption of radial glia and abnormal formation of neocortical layers. Cereb Cortex 9:121-136.

O'Leary DDM, Schlaggar BL, Tuttle R (1994) Specification of neocortical areas and thalamocortical connections. Annu Rev Neurosci $17: 419-439$

Stryker MP, Harris WA (1986) Binocular impulse blockade prevents the formation of ocular dominance columns in cat visual cortex. J Neurosci 6:2117-2133.

Woo TU, Finlay BL (1996) Cortical target depletion and ingrowth of geniculocortical axons: implications for cortical specification. Cereb Cortex 6:457-469.

Yamamoto N, Higashi S, Toyama K (1997) Stop and branch behaviors of geniculocortical axons: a time lapse study in organotypic cocultures. J Neurosci 17:3653-3663.

Yurkewicz L, Valentino KL, Floeter MK, Fleshman Jr JW, Jones EG (1984) Effects of cytotoxic deletions of somatic sensory cortex in fetal rats. Somatosens Res 1:303-327.

Zedeck MS, Brown GB (1977) Methylation of intestinal and hepatic DNA in rats treated with methylazoxymethanol acetate. Cancer 40:2580-2583.

Zedeck MS, Swislocki NI (1975) Effect of methylazoxymethanol acetate on adenylate cyclase and $5^{\prime}$-nucleotidase in rat liver plasma membranes. Cancer Lett 1:109-112.

Zedeck MS, Sternberg SS, Poynter RW, McGowan J (1970) Biochemical and pathological effects of methylazoxy methanol acetate, a potent carcinogen. Cancer Res 30:891-912. 